\title{
Review
}

Neuroepidemiology

\section{COVID-19 Infection and Neurological Complications: Present Findings and Future Predictions}

\author{
Ettore Beghi $^{\mathrm{a}}$ Valery Feigin ${ }^{\mathrm{b}}$ Valeria Caso ${ }^{c}$ Paola Santalucia $^{d}$ \\ Giancarlo Logroscino ${ }^{\mathrm{e}}$
}

aDepartment of Neuroscience, Istituto di Ricerche Farmacologiche Mario Negri IRCCS, Milan, Italy; bational Institute for Stroke and Applied Neurosciences School of Public Health and Psychosocial Studies Faculty of Health and Environmental Sciences AUT University Auckland, Auckland, New Zealand; 'Stroke Unit, Santa Maria della Misericordia Hospital, University of Perugia, Perugia, Italy; ${ }^{d}$ Azienda Sanitaria Provinciale-Ragusa, Ragusa, Italy; eCenter for Neurodegenerative Diseases and the Aging Brain, Department of Clinical Research in Neurology, University of Bari "Aldo Moro"Bari, "Pia Fondazione Cardinale G. Panico", Tricase, Italy

\section{Keywords}

Coronavirus · Neurological disorders · Outbreak · Infection

\begin{abstract}
The present outbreak caused by SARS-CoV-2, an influenza virus with neurotropic potential, presents with neurological manifestations in a large proportion of the affected individuals. Disorders of the central and peripheral nervous system are all present, while stroke, ataxia, seizures, and depressed level of consciousness are more common in severely affected patients. People with these severe complications are most likely elderly with medical comorbidities, especially hypertension and other vascular risk factors. However, postinfectious complications are also expected. Neurological disorders as sequelae of influenza viruses have been repeatedly documented in the past and include symptoms, signs, and diseases occurring during the acute phase and, not rarely, during follow-up. Postinfectious neurological complications are the result of the activation of immune mechanisms and can explain the insurgence of immune-mediated diseases, including the Guillain-Barré syndrome and other diseases of the central and peripheral nervous system that in the past
\end{abstract}

karger@karger.com

(c) 2020 S. Karger AG, Basel

www.karger.com/ned

Karger! occurred as complications of viral infections and occasionally with vaccines. For these reasons, the present outbreak calls for the introduction of surveillance systems to monitor changes in the frequency of several immune-mediated neurological diseases. These changes will determine a reorganization of the measures apt to describe the interaction between the virus, the environment, and the host in areas of different dimensions, from local communities to regions with several millions of inhabitants. The public health system, mainly primary care, needs to be strengthened to ensure that research and development efforts are directed toward right needs and directions. To cope with the present pandemic, better collaboration is required between international organizations along with more research funding, and tools in order to detect, treat, and prevent future epidemics.

(c) 2020 S. Karger AG, Basel

\section{Introduction}

An unexpected infection caused by a coronavirus, SARS-CoV-2, is devastating the health of the world population and, consequently, the global economy. Although 
the primary manifestation of COVID-19 infection is pneumonia, there is increasing evidence that suggests the spread to key organs other than the respiratory system, among them the central and peripheral nervous system. A growing body of evidence shows that neurotropism is one common feature of coronaviruses [1]. The involvement of the nervous system can be due to a direct action of these viruses on the nervous tissue and/or to an indirect action through the activation of immune-mediated mechanisms. While the first action can be verified during the acute phase of the disease, the second can be apparent only after days, weeks, or even months following the acute phase. Many viral infections can damage the structure and function of the nervous system, manifesting as encephalitis, toxic encephalopathy, and postinfectious demyelinating disease [2]. Coronaviruses can invade the nervous tissues involving immune-functioning macrophages, microglia, or astrocytes [3] and cause nerve damage through direct infection pathways (circulatory and neuronal), hypoxia, immune injury, attack to ACE2 enzymes, and other mechanisms [4]. These observations need be considered to provide the background and rationale for the activation of monitoring of affected individuals in the attempt to identify those at risk of developing acute, short-, and long-term neurological complications.

\section{Present Findings on the Association between COVID-19 Infection and the Occurrence of Neurological Disorders}

In one of the first reports on neurological findings during the COVID-19 outbreak in Wuhan, China, neurological manifestations were present in $78 / 214$ patients $(36.4 \%)$ and fell into 3 categories: central nervous system (dizziness, headache, impaired consciousness, acute cerebrovascular disease, ataxia, and seizure), peripheral nervous system (taste impairment, smell impairment, vision impairment, and nerve pain), and muscular-skeletal [5]. Stroke, ataxia, seizures, and depressed level of consciousness were most common in severely affected patients. In a retrospective study of patients admitted in a single center in Wuhan, 11/221 patients with COVID-19 developed acute ischemic stroke, 1 cerebral venous sinus thrombosis, and 1 cerebral hemorrhage. However, patients with these severe complications are more likely to be elderly and present medical comorbidities, especially vascular risk factors such as hypertension [6]. Hypoxic encephalopathy has been diagnosed in $20 \%$ of 113 deceased patients with COVID-19 virus [7]. A 31\% incidence of thrombotic com- plications in ICU patients with COVID-19 infection has been documented in $184 \mathrm{ICU}$ patients with proven COVID-19 pneumonia, of whom 23 died (13\%) [8]. In a meta-analysis of 6 studies in patients with COVID-19, cerebrovascular disease was identified as a risk factor along with hypertension, diabetes, chronic obstructive pulmonary disease, and cardiovascular disease [9]. Other neurological features were observed in severe cases, including confusion, diffuse corticospinal tract signs, and dysexecutive syndrome [10]. A complete assessment of neurological signs is often difficult because of drug-induced neuromuscular blockade or early death. Acute inflammatory demyelinating polyneuropathy [11] and Miller-Fisher syndrome [12] were also observed. The occurrence of cerebrovascular events in patients with arterial hypertension and cardiovascular disease might be related to a direct effect of the infection itself or an inappropriate host response. However, in case reports and small series, the possibility of coincidence cannot be excluded [13].

\section{Lessons from the Past}

Neurological complications as sequelae of influenza infection have been documented for over a century. In an interesting historical review, Henry and coworkers [14] found that in a discussion on influenza in 1919, the British Royal Society of Medicine established an association between neurological disorders and the pandemic and postulated that influenza "attacked more particularly the nerve centres" based on the "very pronounced nerve sequelae observed." In the discussion, the authors argued that the temporal relationship between outbreaks of influenza and cerebrospinal fever (meningitis), poliomyelitis, and polio-encephalitis was more than coincidence. Most importantly, encephalitis lethargica and postencephalitic Parkinsonism have been closely associated with the 1918 flu pandemic. Several other viruses have been then associated with secondary Parkinsonisms. These include Coksackievirus; Japanese encephalitis B, St. Louis, and West Nile viruses; and HIV [15].

\section{Viral Infections and Neurological Complications}

Coronaviruses are not the only viruses associated with neurological complications. Zika virus is an arbovirus transmitted mainly by mosquitos of Aedes species. During the recent outbreak due to Zika virus, serious neurological complications were observed, including micro- 
cephaly, Guillain-Barré syndrome (GBS), and eye infections [16]. Less frequently reported neurological complications include encephalitis/meningoencephalitis, acute disseminated encephalomyelitis, myelitis, cerebrovascular diseases (ischemic infarction and vasculopathy), seizures and encephalopathy, sensory polyneuropathy, and neuronopathy [17].

H1N1 2009 orthomyxovirus affected countries in all 5 continents, with most cases reported in North and South America and Europe, and children and young adults being the most susceptible age-groups [18]. In Central America and the Dominican Republic, H1N1 pandemic was lethal in young adults, pregnant women, and people with preexisting medical conditions [19].

Ebolavirus, from the filovirus family, causes severe hemorrhagic fever with significant epidemic potential and high case-fatality. The 2013-2016 outbreak in West Africa was larger than all previous outbreaks combined, with 28,646 reported cases and 11,323 reported deaths. It was also unique in its geographical distribution and multicountry spread [20]. Many neurological symptoms have been described during the acute phase, including altered mental status, seizures, and meningoencephalitis, among others; survivors also developed neurological sequelae, such as persistent headache and memory loss, and abnormalities on neurological exam [21]. The CNS is the site of hiding of the Ebolavirus and is responsible for relapses during the recovery period [22].

In recent years, several non-polio enteroviruses have emerged as serious public health concerns. These include EV-A71, which has caused epidemics of hand-foot-andmouth disease (HFMD) in Southeast Asia, and EV-D68, which recently caused a large outbreak of severe lower respiratory tract disease in North America [23]. HFMD is associated with acute neurological disease in children and a substantial burden of long-term neurological sequelae [24]. Infections due to these viruses are associated with severe neurological complications, including, in addition to HFMD, aseptic meningitis, encephalitis, acute flaccid paralysis, and acute flaccid myelitis.

\section{Other Coronaviruses and Neurological Disorders}

Severe acute respiratory syndrome (SARS) is a zoonotic respiratory disease caused by a coronavirus (SARS$\mathrm{CoV}$ ) starting in Southeast Asia and spreading to other countries in 2003. SARS-CoV could induce several neurological diseases, including polyneuropathy, encephalitis, and aortic ischemic stroke [25]. Autopsy studies dem- onstrated that signs of cerebral edema and meningeal vasodilation could be detected in most cases of SARS. Furthermore, infiltration of monocytes and lymphocytes in the vessel wall, ischemic changes of neurons, demyelination of nerve fibers, and SARS-CoV particles and genome sequences could be detected in the brain [26].

Another zoonotic disease is the Middle East respiratory syndrome (MERS) that in 2012 marked the introduction of MERS-CoV, another highly pathogenic coronavirus introduced into the human population. MERS- $\mathrm{CoV}$ is also known to be potentially neuroinvasive, with $25.7 \%$ of patients developing insanity and $8.6 \%$ experiencing seizures [27]. Neurological symptoms occurring during the infection process also included disturbance of consciousness, paralysis, ischemic stroke, GBS, and other poisoning or infectious neuropathies [28]. Seropositivity for coronaviruses has been reported in a variety of neurological disorders, including encephalitis [29], optic neuritis [30], multiple sclerosis [31], and Parkinson's disease [32].

\section{Neurological Complications: Direct Viral Effects versus Adverse Effects of Vaccines}

A significantly increased incidence of GBS after swine flu vaccination in the USA in 1976 led to a debate on the possible link between influenza vaccinations and GBS [33]. The reported relative risk was 7.6 corresponding to about ten excess cases of GBS per million vaccinations. Between 1978 and 2009, several studies on the association between influenza vaccination and GBS yielded conflicting results [34]. Although the biological mechanisms remain unsettled [35], a study in mice [36] suggested that influenza vaccine antigens may induce cross-reactive anti-ganglioside antibodies, eventually causing peripheral nerve damage. During the influenza pandemic in 2009, the possible link between influenza vaccination and GBS drew special attention due to the rapid development and implementation of vaccines against pandemic influenza $\mathrm{A} / \mathrm{H} 1 \mathrm{~N} 1$ virus. A case-control study was conducted in Italy between October 2010 and May 2011 to explore the association between influenza vaccination and GBS [34]. Influenza vaccination was associated with GBS, with a relative risk of 2.1 (95\% CI 1.1, 3.9), giving an attributable risk from 2 to 5 GBS cases per 1,000,000 vaccinations. These findings were in keeping with the results of eight studies conducted during the 2009-2010 influenza vaccine campaign with a monovalent $\mathrm{A} / \mathrm{H} 1 \mathrm{~N} 1$ vaccine, which showed a non-significant or at best small increase of the risk [34]. The results of those studies do not mod- 
ify the risk-benefit profile of seasonal influenza vaccination but call attention to the need to develop a vaccine with a favorable safety profile.

\section{True Neurological Complications or Stochastic Findings?}

The increased incidence of GBS after swine flu vaccination raised several claims that the vaccine was the cause of several other infectious or immune-mediated diseases, including postvaccinal encephalopathy, myelopathy, and peripheral nerve lesions [37]. These claims led the US government to ask Leonard T. Kurland, an eminent US neuroepidemiologist from the Mayo Clinic in Rochester, Minnesota, USA, to provide reference data on the incidence of a number of infectious and immune-mediated inflammatory neurological diseases, including encephalitis and aseptic meningitis [38], acute transverse myelitis [39], brachial plexus neuropathy [40], and GBS [41], and calculate the excess number of individuals who could experience the same symptoms as a result of the adverse effects of the vaccine. This interesting experience provides the background for correct investigation of the measure of the risk in the presence of a possible association between an etiological agent (in our case, the COVID-19) and a neurological disease.

\section{Problems Arising When Assessing a Cause-Effect Relationship between the Virus and Acute and Long-Term Complications}

The unexpected clinical manifestations of the COVID-19 outbreak led the medical community and the society at large to react in differing ways and introduce variable contention measures that interfered with a correct assessment of the cause-effect relationship between the extent of contagion, development of symptoms, disease severity and complications, and outcome. These measures included, among others, (1) the variable control of the environment and the local setting (workplace, transports, amusement places, healthcare facilities, nursing homes, etc.); (2) the uncontrolled use of RT-PCR-based assays on respiratory specimens, some of them with still unproven validity and reliability; and (3) the variable measures introduced to reduce social contacts. These non-standardized medical interventions must be contrasted with the intrinsic heterogeneity of the interaction of viral infection and host reaction, which includes (1) the reproductive number; (2) duration of the incubation period; (3) demographic and clinical characteristics of the patient (age, sex, comorbidities, and immune status); and, not least, (4) the possible adverse effects of treatments and treatment categories. Thus, the insurgence of a neurological disease in the acute phase of the infection must be assessed in the light of this complex scenario, in order to verify whether the disease is the direct effect of the viral aggression (meningitis, encephalitis, or acute encephalopathy) or an indirect, immune-mediated, effect. Compared to the former, the latter is more difficult to investigate and requires the calculation of the number of cases that during follow-up might be expected in the area due to the incidence of that same disease in the general population.

\section{Preventive Measures and Treatments}

The generalized fear provoked by the pandemic might have significant reflections on several neurological disorders in terms of diagnostic assessment and overall management. Symptoms perceived by patients and/or caring physicians as not requiring immediate neurological consultation might have delayed a timely diagnosis and proper treatment. This might have occurred for people with stroke $[42,43]$ and can be expected for other conditions requiring hospitalization. The chaotic management of patients considered to be at risk of contagion or who presented with general or neurological manifestations of infection had perhaps a negative impact on the introduction of preventive and therapeutic measures. This includes, among others, the measurement of D-dimer at the onset of the infection to prevent thrombosis [44]. Last, the uncontrolled use of several drugs without waiting for the results of properly designed and conducted trials might expose the patients to unpredictable benefits at the expense of previously documented adverse effects.

\section{Future Directions}

The information available on the frequency of neurological disorders in people with COVID-19 is entirely based on clinical series that, in the absence of controls and a population base, give us only a rough and perhaps biased picture of the purported association and do not provide clues on the direction. In addition, we cannot predict if this association ends with the resolution of the outbreak or, most likely, will be followed by a number of short- 
term and long-term complications. The picture is further complicated by the poor knowledge of the seasonal variation of the infection, the duration of immunity, the intensity of cross-immunity, and the effects of control measures [45]. As taught by the 1918 influenza pandemic and the more recent occurrence of postinfectious neurological complications of coronaviruses, present observations provide the basis to implement surveillance programs directed at the detection of selected neurological disorders as epidemiological markers of immune-mediated reactions to the virus. GBS might be selected as one of these disorders considering that the incidence of the disease has been calculated in several countries in all continents [46].

The Institute of Health Metrics and Evaluation, site of the Global Burden of Disease (GBD) Collaboration, has developed projections of resource utilization in response to the COVID-19 pandemic [47]. The projected estimates are based on modeling the peak in death rates and hospital usage in Wuhan city in China, where the virus was first discovered, and data from the US and several European countries. The GBD Collaboration might provide the background information against which the burden of neurological disorders at the time of the outbreak will be contrasted in order to assess the geographic and temporal trends of this burden at a global level.

A registry promoted by the European Academy of Neurology and aimed at defining the spectrum of neurological manifestations during and after the COVID-19 outbreak in various European countries is in preparation. This registry, in addition to several ongoing national and local surveillance programs, might help at least define the burden of neurological disorders that come to medical attention.

The lessons learned from previous and current observations of the impact of pandemics on neurological manifestations and global health emphasize the need for strengthening health systems, to ensure that research and development efforts are directed toward existing needs.
People with neurological disorders might be at risk of incurring the most severe complications of the infectious disease, and, conversely, the infection itself might be a source of neurological complications. These observations have profound reflections on the management of acute neurological conditions like stroke and chronic conditions like multiple sclerosis, neurodegenerative disorders, and myasthenia gravis. Furthermore, the implementation of social distancing and interruption of all nonurgent clinical activities, thus limiting access to hospitals, have induced significant problems in the clinical care of several chronic neurological conditions. The likely worsening of the outcomes of these neurological conditions due to the change in access to medical care is part of the impact of COVID-19 infection that should be measured [48].

Curbing the impact of future viral epidemics, or comparable diseases, requires increased long-term investments in health system strengthening, better collaboration between different international organizations, more funding for research, and tools to detect, treat, and prevent future epidemics.

\section{Disclosure Statement}

The authors have no disclosures and no conflicts of interest to declare with reference to this topic.

\section{Funding Sources}

The authors did not receive any funding.

\section{Author Contributions}

E.B. conceived the review and drafted the manuscript; G.L. added important concepts and revisions; all the other authors revised the text, providing significant intellectual contribution.

\section{References}

1 Li YC, Bai WZ, Hashikawa T. The neuroinvasive potential of SARS-CoV2 may play a role in the respiratory failure of COVID-19 patients. J Med Virol. 2020 Feb 27.

2 Michalicová A, Bhide K, Bhide $M$, Kováč A. How viruses infiltrate the central nervous system. Acta Virol. 2017;61(04):393-400.

3 Al-Obaidi MMJ, Bahadoran A, Wang SM, Manikam R, Raju CS, Sekaran SD. Disruption of the blood brain barrier is vital property of neurotropic viral infection of the central nervous system. Acta Virol. 2018;62(1):16-27.
4 Wu Y, Xu X, Chen Z, Duan J, Hashimoto K, Yang L, et al. Nervous system involvement after infection with COVID-19 and other coronaviruses. Brain Behav Immun. 2020 Mar 30; S0889-1591(20)30357-3.

5 Mao L, Jin H, Wang M, Hu Y, Chen S, He Q, et al. Neurologic manifestations of hospitalized patients with coronavirus disease 2019 in Wuhan, China. JAMA Neurol. 2020 Apr 10; e201127.
6 Guan WJ, Ni ZY, Hu Y, Liang WH, Ou CQ, $\mathrm{He}$ JX, et al. Clinical characteristics of coronavirus disease 2019 in China. N Engl J Med. 2020 Apr 30;382(18):1708-20. http://dx.doi. org/10.1056/NEJMoa2002032.

7 Chen T, Wu D, Chen H, Yan W, Yang D, Chen G, et al. Clinical characteristics of 113 deceased patients with coronavirus disease 2019: retrospective study. BMJ, 2020 Mar 26; 368:m1091. Erratum in: BMJ. 2020 Mar 31; 368:m1295. 
8 Klok F, Kruip M, van der Meer N, Arbous M, Gommers D, Kant K, et al. Incidence of thrombotic complications in critically ill ICU patients with COVID-19. Thromb Res. 2020 Jul;191:145-7. 10.1016/j.thromres.2020. 04.013 .

9 Wang B, Li R, Lu Z, Huang Y. Does comorbidity increase the risk of patients with $\mathrm{CO}$ VID-19: evidence from meta-analysis. Aging. 2020 Apr;812(7):6049-57. http://dx.doi.org/ 10.18632/aging. 103000.

10 Helms J, Kremer S, Merdji H, Clere-Jehl R, Schenck M, Kummerlen C, et al. Neurologic features in severe SARS-CoV-2 infection. N Engl J Med. 2020 Jun;4382(23):2268-70. http: //dx.doi.org/10.1056/nejmc2008597.

11 Toscano G, Palmerini F, Ravaglia S, Ruiz L, Invernizzi P, Cuzzoni MG, et al. Guillain-Barré syndrome associated with SARS-CoV-2. N Engl J Med. 2020 Apr 17. Epub ahead of print.

12 Gutiérrez-Ortiz C, Méndez A, Rodrigo-Rey S, San Pedro-Murillo E, Bermejo-Guerrero L, Gordo-Mañas R, et al. Miller Fisher syndrome and polyneuritis cranialis in COVID-19. Neurology. 2020 Apr 17; 10.1212/WNL. 0000000000009619. doi: 10.1212/WNL. 0000000000009619. Epub ahead of print.

13 Zhao H, Shen D, Zhou H, Liu J, Chen S. Guillain-Barré syndrome associated with SARSCoV-2 infection: causality or coincidence? Lancet Neurol. 2020;19(5):383-4. 10.1016/ S1474-4422(20)30109-5.

14 Henry J, Smeyne RJ, Jang H, Miller B, Okun MS. Parkinsonism and neurological manifestations of influenza throughout the 20th and 21st centuries. Parkinsonism Relat Disord. 2010;16(9):566-71.

15 Jang H, Boltz DA, Webster RG, Smeyne RJ. Viral parkinsonism. Biochim Biophys Acta. 2009;1792(7):714-21.

16 Koppolu V, Shantha Raju T. Zika virus outbreak: a review of neurological complications, diagnosis, and treatment options. J Neurovirol. 2018;24(3):255-72.

17 Carod-Artal FJ. Neurological complications of Zika virus infection. Expert Rev Anti Infect Ther. 2018;16(5):399-410.

18 Reddy D. Responding to pandemic (H1N1) 2009 influenza: the role of oseltamivir. J Antimicrob Chemother. 2010;65(Suppl 2):ii3540.

19 Chacon R, Mirza S, Rodriguez D, Paredes A, Guzman G, Moreno L, et al. Demographic and clinical characteristics of deaths associated with influenza $\mathrm{A}(\mathrm{H} 1 \mathrm{~N} 1)$ pdm09 in Central America and Dominican Republic 20092010. BMC Public Health. 2015;15:734.

20 Coltart CE, Lindsey B, Ghinai I, Johnson AM, Heymann DL. The Ebola outbreak, 20132016: old lessons for new epidemics. Philos Trans R Soc Lond, B, Biol Sci. 2017;372(1721): 20160297.

21 Billioux BJ. Neurological complications and sequelae of Ebola virus disease. Curr Infect Dis Rep. 2017;19(5):19.
22 Wong G, Qiu X, Bi Y, Formenty P, Sprecher $\mathrm{A}$, Jacobs $\mathrm{M}$, et al. More challenges from Ebola: infection of the central nervous system. J Infect Dis. 2016;214(Suppl 3):S294-6.

23 Baggen J, Thibaut H, Strating J, van Kuppeveld $F$. The life cycle of non-polio enteroviruses and how to target it. Nat Rev Microbiol. 2018; 16(6): 368-81. 10.1038/s41579-0180022-3.

24 Jones E, Pillay TD, Liu F, Luo L, Bazo-Alvarez JC, Yuan C, et al. Outcomes following severe hand foot and mouth disease: a systematic review and meta-analysis. Eur J Paediatr Neurol. 2018;22(5):763-73.

25 Tsai L, Hsieh S, Chang Y. Neurological manifestations in severe acute respiratory syndrome. Acta Neurol Taiwan. 2005;14(3):1139. 16252612 .

$26 \mathrm{Gu}$ J, Gong E, Zhang B, Zheng J, Gao Z, Zhong $\mathrm{Y}$, et al. Multiple organ infection and the pathogenesis of SARS. J Exp Med. 2005; 202(3):415-24.

27 Saad M, Omrani AS, Baig K, Bahloul A, Elzein F, Matin MA, et al. Clinical aspects and outcomes of 70 patients with Middle East respiratory syndrome coronavirus infection: a single-center experience in Saudi Arabia. Int J Infect Dis. 2014;29:301-6.

28 Kim JE, Heo JH, Kim HO, Song SH, Park SS, Park TH, et al. Neurological complications during treatment of Middle East respiratory syndrome. J Clin Neurol. 2017;13(3):227-33.

29 Li Y, Li H, Fan R, Wen B, Zhang J, Cao X, et al. Coronavirus infections in the central nervous system and respiratory tract show distinct features in hospitalized children. Intervirology. 2016;59(3):163-9.

30 Dessau R, Lisby G, Frederiksen J. Coronaviruses in spinal fluid of patients with acute monosymptomatic optic neuritis. Acta Neurol Scand. 1999;100(2):88-91.10.1111/j.16000404.1999.tb01043.x.

31 Salmi A, Ziola B, Hovi T, Reunanen M. Antibodies to coronaviruses OC43 and 229E in multiple sclerosis patients. Neurology. 1982; 32(3):292-5.

32 Fazzini E, Fleming J, Fahn S. Cerebrospinal fluid antibodies to coronavirus in patients with Parkinson's disease. Mov Disord. 1992; 7(2):153-8

33 Schonberger LB, Bregman DJ, Sullivan-Bolyai JZ, Keenlyside RA, Ziegler DW, Retailliau HF, et al. Guillain-Barre syndrome following vaccination in the National Influenza Immunization Program, United States, 1976-1977. Am J Epidemiol. 1979;110(2):105-23.

34 Galeotti F, Massari M, D’Alessandro R, Beghi E, Chiò A, Logroscino G, et al. Risk of Guillain-Barré syndrome after 2010-2011 influenza vaccination. Eur J Epidemiol. 2013; 28(5):433-44.

35 Sejvar JJ, Baughman AL, Wise M, Morgan OW. Population incidence of Guillain-Barré syndrome: a systematic review and metaanalysis. Neuroepidemiology. 2011;36(2): 123-33.
36 Nachamkin I, Shadomy SV, Moran AP, Cox $\mathrm{N}$, Fitzgerald $\mathrm{C}$, Ung $\mathrm{H}$, et al. Anti-ganglioside antibody induction by swine (A/NJ/1976/ H1N1) and other influenza vaccines: insights into vaccine-associated Guillain-Barré syndrome. J Infect Dis. 2008;198(2):226-33.

37 Poser C. Neurological complications of swine influenza vaccination. Acta Neurol Scand.1982;66(4):413-31.10.1111/j.1600-0404. 1982.tb06864.x.

38 Beghi E, Nicolosi A, Kurland LT, Mulder DW, Hauser WA, Shuster L. Encephalitis and aseptic meningitis, Olmsted County, Minnesota, 1950-1981: I. Epidemiology. Ann Neurol. 1984;16(3):283-94.

39 Beghi E, Kurland LT, Mulder DW. Incidence of acute transverse myelitis in rochester, Minnesota, 1970-1980, and implications with respect to influenza vaccine. Neuroepidemiology. 1982;1(3):176-88.

40 Beghi E, Kurland LT, Mulder DW, Nicolosi A. Brachial plexus neuropathy in the population of Rochester, Minnesota, 1970-1981. Ann Neurol. 1985;18(3):320-3.

41 Beghi E, Kurland LT, Mulder DW, Wiederholt WC. Guillain-Barré syndrome. Clinicoepidemiologic features and effect of influenza vaccine. Arch Neurol. 1985;42(11):1053-7.

42 Naccarato M, Scali I, Olivo S, Ajčević M, Buoite Stella A, Furlanis G, et al. Has COVID-19 played an unexpected "stroke" on the chain of survival? J Neurol Sci. 2020 May 6; 414:116889. Epub ahead of print.

43 Caso V, Federico A. No lockdown for neurological diseases during COVID19 pandemic infection. Neurol Sci. 2020;41(5):999-1001. 10.1007/s10072-020-04389-3.

44 Kermali M, Khalsa RK, Pillai K, Ismail Z, Harky A. The role of biomarkers in diagnosis of COVID-19: a systematic review. Life Sci. 2020 May 13;254:117788. Online ahead of print.

45 Kissler SM, Tedijanto C, Goldstein E, Grad $\mathrm{YH}$, Lipsitch M. Projecting the transmission dynamics of SARS-CoV-2 through the postpandemic period. Science. 2020 May 22; 368(6493): 860-8. doi: 10.1126/science. abb5793.

46 Sejvar JJ, Pfeifer D, Schonberger LB. Guillainbarré syndrome following influenza vaccination: causal or coincidental? Curr Infect Dis Rep. 2011;13(4):387-98.

47 IHME COVID-19 health service utilization forecasting team. Forecasting the impact of the first wave of the COVID-19 pandemic on hospital demand and deaths for the USA and European Economic Area countries. Submitted April 21, 2020 as a preprint to the medRxiv server as MEDRXIV/2020/074732.

48 Capozzo R, Zoccolella S, Musio M, Barone R, Accogli M, Logroscino G. Telemedicine is a useful tool to deliver care to Amyotrophic Lateral Sclerosis patients during COVID-19 pandemic: results from Southern Italy. ALS \& FTD. 2020. In press. 